\title{
A WAVELET-BASED APPROACH TO MONITORING PARKINSON'S DISEASE SYMPTOMS
}

\author{
Avishai Wagner Naama Fixler $\quad$ Yehezkel S. Resheff \\ Intel Advanced Analytics
}

\begin{abstract}
Parkinson's disease is a neuro-degenerative disorder affecting tens of millions of people worldwide. Lately, there has been considerable interest in systems for at-home monitoring of patients, using wearable devices which contain inertial measurement units. We present a new wavelet-based approach for analysis of data from single wrist-worn smart-watches, and show high detection performance for tremor, bradykinesia, and dyskinesia, which have been the major targets for monitoring in this context. We also discuss the implication of our controlled-experiment results for uncontrolled home monitoring of freely behaving patients.
\end{abstract}

Index Terms - Parkinson's Disease, Monitoring, Wearable Devices, Accelerometer, Wavelets

\section{INTRODUCTION}

Parkinson's Disease (PD) is a neuro-degenerative disorder affecting tens of millions of people worldwide [1]. PD is associated with motor symptoms such as tremor, rigidity, bradykinesia and gait impairments. Another motor difficulty is Levodopa induced dyskinesia - a side effect of chronic Levodopa therapy, characterized by involuntary and unorganized movement. Currently, the accepted clinical measurement of PD symptom severity is the Unified Parkinsons Disease Rating Scale (UPDRS), which is based, in part, on subjective and potentially recall-distorted reports by the patients, and on semiobjective observations by the clinician [2]. The UPDRS is typically assessed merely two to three times a year (when the clinician meets the patient face to face), and therefore tends to miss occasional fluctuations in the state of the patients. These fluctuations are a very common phenomenon in PD. The lack of measure continuity and objectivity allows for variation and potential biases in diagnoses or determinations of PD-related motor states. This may adversely affect the prescribed treatment plan.

Partially due to the limitations of the UPDRS standard, PD has been an early target for experimentation with monitoring using wearable devices which contain inertial measurement units [3]. Replacing subjective patient self-reports with objective measures may improve and standardize the way physicians understand the actual state of the disease. In addition, long-term monitoring of symptoms will allow a system to detect and flag changes requiring intervention, thus improving the care patients are able to receive. Finally, longitudinal continuous collection of clinical objective measures from a large cohort can reduce clinical trial costs and duration, and accelerate research in the field of PD.

There have been several attempts to design systems for PD monitoring with wearable devices. MercuryLive [4, 5] is a system for at-home monitoring of PD patients, using a network of SHIMER sensors [6] to monitor symptoms and activity. Kinesia system [7] is comprised of a single 12 gram finger worn sensor and an 85 gram wrist worn computing module, connected via a wire. The predicted tremor score, output by this system, was shown to correlate with clinical scores [8]. Although demonstrating the ability to extract some significant objective measures from wearable sensors, the equipment used in these systems poses significant scalability limitations, due to its cost, complexity and burden to the patient.

An efficient and usable monitoring system should include a single wearable element [9]; if possible, a standard unobtrusive device. Few researchers explored the potential of using a consumer smartphone as a measurement device for PD based on a set of predefined tests [10]. This approach requires a significant commitment from the patient, and doesn't necessarily reflect natural activities of daily living.

Previously, analysis of accelerometer signals for symptom prediction [11] or behavior detection [12] has mostly been done using hand-crafted features, suitable for the task at hand, and demonstrated on data collected in highly controlled environments. The main contribution of this paper is in the novel wavelet-based feature engineering approach, in the context of PD patient monitoring. We show that our method leads to high accuracy classification of multiple PD-related symptoms, using data from single wrist-worn smart-watches. Our method is appropriate for long term at-home monitoring of freely behaving individuals, as a long-term, unobtrusive, wearable solution.

\section{DATA COLLECTION}

Data was collected from 19 PD patients (age: $62 \pm 8.8$ years; disease duration: $8 \pm 4.5$ years), during two days of hospital visits, and two additional days of home monitoring. During each visit the patient preformed 20 motor tasks. Each task was repeated 6 times in each visit, resulting in 240 task segments 
per patient.

Motor tasks performed by the patients were divided into 5 general groups of activity: Resting (e.g. sitting quietly), gross upper limb movement (e.g. folding towels), fine upper limb (e.g. drawing), periodic hand movement (e.g. hand rotation), and walking.

During each task, a physician graded a number of symptoms (tremor, dyskinesia, bradykinesia) on a score from 0 to 4 , where 0 indicates the symptom is not present, and 4 indicates a strong presence. For modeling purposes, we grouped scores 2, 3 and 4 into one bin, due to imbalanced distribution of the values. We treat the labels as ground truth while being aware of the inter-rater variability associated with such tests, and its implications on the quality of automatic classification.

Subjects arrived at the clinic twice, in different stages of the medication effect cycle, to allow observation of the same patients with differential symptom severity. The first visit started in the on state during which the patient experienced a positive response to medication, and continued through the transitioning to the off state, during which the patient experienced a reemergence of the Parkinsonian symptoms suppressed during the "on" state. The second visit started in the clinical off state, following at least 12 hours without medication intake, and following medication intake, continued through the transitioning of the subject to the on state.

Raw tri-axial accelerometer data was collected using a single wrist-worn smart-watch (GENEActiv), at $50 \mathrm{~Hz}$ throughout the visit, including the full duration of the motor tasks and the breaks between them in which the patients were free to behave naturally. During the two days of home monitoring, accelerometer data was recorded continuously at $50 \mathrm{~Hz}, 24$ hours a day.

\section{MODELING}

\subsection{Wavelet features extraction}

The wavelet transform has proven to be an effective tool in signal processing, classification, and clustering [13, 14]. Wavelets can handle non-stationary signals better than the Fourier transform, and capture localized information on the time-frequency plane. This property is very important in our case, since we are trying to capture events in time and frequency such as temporary tremor. The wavelet coefficients are obtained by translating and scaling a unique mother wavelet function. For a signal of length $2^{k}$, we have in total $k$ scales, where each scale represent a different level of frequencies, with higher scales relating to the lower frequency. Using signals of a length which is a power of 2 allows us to make use of the pyramidal algorithm [15] to obtain the wavelet coefficients at different scales.

Following [13], we use the absolute and relative contribution of each scale. Specifically, denoting the contribution to

\footnotetext{
${ }^{1}$ www.geneactiv.org
}

the total energy, of each scale cont $_{j}$, we then use the relative energy:

$$
\operatorname{rel}_{j}=\operatorname{cont}_{j} / \sum_{i}\left(\text { cont }_{i}\right)
$$

For a signal of length $2^{k}$ we now have in total $2 k$ features, $\mathrm{k}$ contribution features and $\mathrm{k}$ relative energy features. These features have the advantage of being very easy to compute, helpful in understanding the dominant frequencies in our signal, and as we show, very useful for PD symptoms detection.

\subsection{Symptoms estimation}

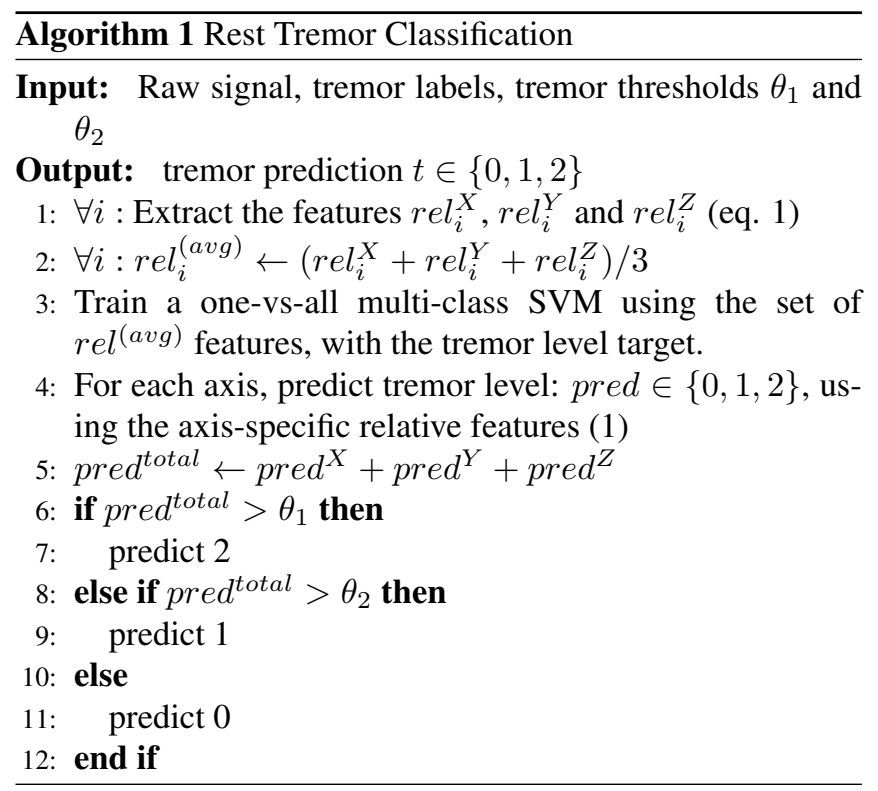

For each axis $(\mathrm{x}, \mathrm{y}, \mathrm{z})$ both relative energy features (equation 11) and mean relative energy (average over the 3 axes, of the former) are computed for each of the wavelet scales. During training, the mean relative energy features are used in a Support Vector machine (SVM) to predict the tremor level (0, 1 , or 2) as annotated by the physician during the task.

Next, the model is utilized to evaluate a per-axis tremor score by using the axis-specific relative energy features in the trained SVM. Finally, the sum of the per-axis scores is threshold-ed heuristically to obtain the final prediction (see Algorithm 11). The rational behind this approach is that while the tremor manifests often in only a single axis, the physician provides a single score which is then assigned to the data from all three axes. The final stage may be improved by using a more rigorous machine learning approach, and learning a function from the per-axis scores to the final classification.

The model described above in Algorithm 1 is used for the prediction of tremor in resting conditions. The same method is used to predict bradykinesia in gross upper limbs movements, and dyskinesia in rest (both are binary classifications 


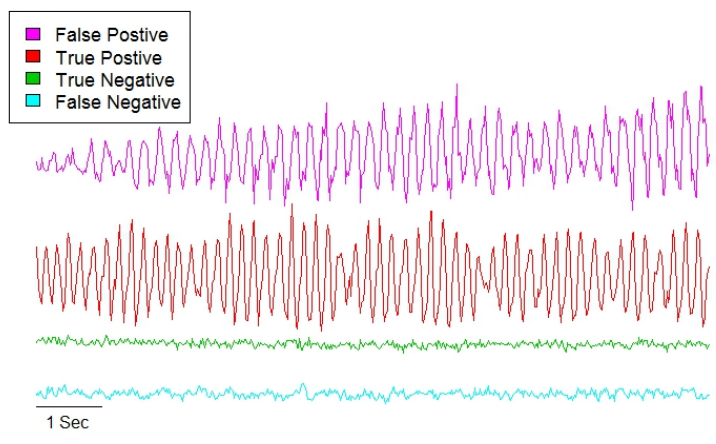

Fig. 1: Misclassification or mislabeling? Raw accelerometer signals from patients at rest with and without tremor. Visually similar signals were labeled differently by the physician.

and thus the final decision in rows 6-11 is replaced with a single threshold). We omit the full description of these models due to space considerations.

\section{EXPERIMENTS AND RESULTS}

\subsection{Data processing}

Raw accelerometer data was split into 10 second intervals (500 samples), with 50\% (5 second) overlap between adjacent segments to ensure that momentary patterns to be detected are not missed because of boundary effects. Since the duration of many of the motor tasks is longer than 10 seconds, each interval was assigned the scores associated with the task segment it belonged to. The 10 second intervals were then further processed using a wavelet transform, as described in Section 3.1.

In order to obtain a signal windows of a length which is a power of 2, we use a spline interpolation (in the output we have is a signal with the length $2^{k}$ where $\mathrm{k}$ is the smallest integer such that the length of the signal is smaller than $2^{k}$ ). We then apply the Debuchy wavelet transform [16] on the output and use the relative contribution features as described in Section 3.1.

\subsection{Evaluation}

All models were evaluated using leave one patient out crossvalidation; a 19-fold cross validation, where each group is the the set of intervals which belong to one of the 19 patients. This procedure helps avoid over-fitting our models to specific patients, allowing the models to have global value, and good generalization properties for new patients.

\subsection{Tremor}

Tremor is among the more visible symptoms of PD, and arguably the most associated with it. Figure 1 shows typical

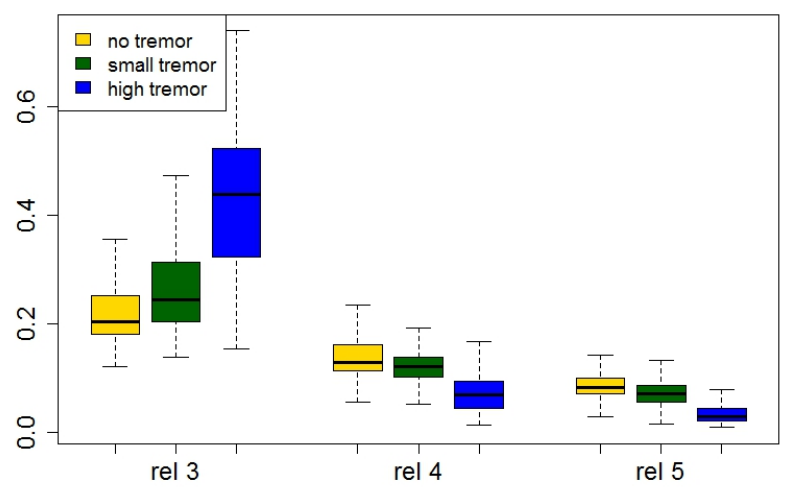

Fig. 2: Boxplots of the 3, 4, and 5 relative scales, for each tremor level, as assigned by a physician.

signals from a patient at rest labeled by a physician as with or without tremor (second and third lines respectively).

Rest tremor is characterized by a fast oscillation of the accelerometer signal, making the relative energy features most appropriate for classification of the extent of tremor $(0,1$, or 2 labels correspond to no-tremor, some-tremor and severetremor). Figure 2 shows that the higher frequencies such as $\mathrm{rel}_{3}$ are much more pronounced in strong tremor, than the lower frequencies such as $\mathrm{rel}_{4}, \mathrm{rel}_{5}$.

The results of our classification model for rest tremor are displayed in Table 1 1. The main source of error is in separating no tremor at all (0 label) and very small tremor such as finger tremor or tremor that is suppressed by the patient (1 label), in both cases the tremor is apparently not detected by the sensor. Moreover, some of our algorithm's mistakes are probably labeling mistakes (see Fig. 1 for representative examples).

We stress that the suggested method is very convenient for use in home-monitoring environments. The data required is derived solely from a wrist-worn smart-watch which does not overly burden the patient, and the model uses fast and standard calculations suitable for real-time systems on any platform.

\subsection{Dyskinesia}

\subsubsection{Rest dyskinesia}

Dyskinesia is a motor problem involving involuntary movements, and is a known side-effect of long term PD levodopa therapy, affecting up to $80 \%$ of chronically treated patients [17]. During rest, we expect these involuntary movements of the body to manifest in a significantly lower frequency band than tremor.

The binary classifier for this task (Dyskinesia present or not) utilizes both absolute and relative energy features. Data from signal segments where the patient is either sitting or standing are used. We modify the algorithm for tremor classi- 
fication (Algorithm 1) first by using the two kinds of features, and furthermore, since this is now a binary classification we change the thresholding (lines 6-12) to reflect this. A segment is classified as positive if at least one of the axes received a positive label. An ROC curve was computed by averaging the per-axis probability derived from an SVM, leading to an AUC of $75 \%$.

\subsubsection{Walking dyskinesia}

While we can detect dyskinesia in rest, the task of monitoring strong dyskinesia in active individuals is substantially more complicated since intentional and involuntary movements overlap both in frequency bands and in structure. Yet, for a known well-structured activity, this is still possible.

During regular walking, the majority of the accelerometer signal is limited to a single axis (we denote this the $\mathrm{X}$ axis). We compute for each energy level the relative contribution of the $\mathrm{X}$ axis, relative to the other two axes (see section 4.1 for the definition of the cont $_{i}^{\text {axis }}$ features) as follows:

$$
\forall i: w_{i}=\frac{\operatorname{cont}_{i}^{y} \operatorname{cont}_{i}^{z}}{\operatorname{cont}_{i}^{x}}
$$

Here again we use an SVM with 19-fold cross validation. The AUC in a leave one-patient out cross-validation obtained for this classification is 0.92 (see confusion matrix in Table 1.).

\subsection{Bradykinesia}

Much work has been done on detecting bradykinesia from hand rotation and other periodic hand movements [18]. Here we attempt to monitor bradykinesia in gross upperlimb movements such as folding towels, organizing sheets of paper in a folder, and reaching out to take a glass of water and drinking it. These more natural behaviors are intended to approximate at-home monitoring of patients during everyday activities. In this task, we again use the relative features in the classification process. The AUC for the ROC obtained is $70 \%$.

\section{CONCLUSION}

PD is a prevalent neurodegenerative disease, which manifests predominantly as a movement disorder. As such, it is an interesting use-case for the introduction of wearable devices and sensors as a means of cheap and continuous monitoring of patient condition.

We present here a novel wavelet-based method for calculating objective measures related to the major Parkinsonian symptoms, and monitoring targets: tremor, dyskinesia and bradykinesia. Whereas previous work focused on handcrafting specific features for classification of the various symptoms of interest, the approach adopted here allows the same
Table 1: Confusion matrices for classification of tremor, dyskinesia, and bradykinesia.

(a) resting tremor

\begin{tabular}{|c|c|c|c|}
\hline True/Pred & 0 & 1 & 2 \\
\hline \hline 0 & 1531 & 28 & 8 \\
\hline 1 & 390 & 159 & 10 \\
\hline 2 & 22 & 52 & 218 \\
\hline
\end{tabular}

(b) resting dyskinesia

\begin{tabular}{|c|c|c|}
\hline True/Pred & 0 & 1 \\
\hline \hline 0 & 1264 & 4 \\
\hline 1 & 150 & 123 \\
\hline
\end{tabular}

(c) walking dyskinesia

\begin{tabular}{|c|c|c|}
\hline True/Pred & 0 & 1 \\
\hline \hline 0 & 2244 & 19 \\
\hline 1 & 145 & 309 \\
\hline
\end{tabular}

(d) gross upper limb bradykinesia

\begin{tabular}{|c|c|c|}
\hline True/Pred & 0 & 1 \\
\hline \hline 0 & 2177 & 201 \\
\hline 1 & 340 & 311 \\
\hline
\end{tabular}

simple and systematic features to be applied in the models pertaining to all of the symptoms. We show high performance in terms of detecting symptoms and severity using a single wrist-worn smart-watch.

The central limitation of the current approach is that a single wrist-mounted sensor will inherently miss symptom related movements which occur solely in other parts of the body. We intend to overcome this issue in the future by aggregating symptom predictions over time, thus in effect waiting long enough for the characteristic movement to appear in the limb we are able to measure from.

An important goal of this research is to inspire wearable technology adoption outside of the laboratory setting. Indeed, our results demonstrate that resting tremor can be detected at relatively high accuracy, a finding that likely translates well to the home environment. Future work will additionally focus on evaluation of increasingly native environments towards achieving long term monitoring of PD patients in their daily lives.

\section{ACKNOWLEDGMENTS}

This work is part of the collaboration between the Advanced Analytics health team at Intel and the Michael J. Fox Foundation for Parkinsons disease research. We thank Zeev Waks and Tom Hope for insightful discussions about this work.

\section{REFERENCES}

[1] Theo Vos, Ryan M Barber, Brad Bell, Amelia BertozziVilla, Stan Biryukov, Ian Bolliger, Fiona Charlson, Adrian Davis, Louisa Degenhardt, Daniel Dicker, et al., 
"Global, regional, and national incidence, prevalence, and years lived with disability for 301 acute and chronic diseases and injuries in 188 countries, 1990-2013: a systematic analysis for the global burden of disease study 2013," The Lancet, vol. 386, no. 9995, pp. 743$800,2015$.

[2] Christopher G Goetz, Barbara C Tilley, Stephanie R Shaftman, Glenn T Stebbins, Stanley Fahn, Pablo Martinez-Martin, Werner Poewe, Cristina Sampaio, Matthew B Stern, Richard Dodel, et al., "Movement disorder society-sponsored revision of the unified parkinson's disease rating scale (mds-updrs): Scale presentation and clinimetric testing results," Movement disorders, vol. 23, no. 15, pp. 2129-2170, 2008.

[3] Walter Maetzler, Josefa Domingos, Karin Srulijes, Joaquim J Ferreira, and Bastiaan R Bloem, "Quantitative wearable sensors for objective assessment of parkinson's disease," Movement Disorders, vol. 28, no. 12, pp. 1628-1637, 2013.

[4] Bor-Rong Chen, Shyamal Patel, Thomas Buckley, Ramona Rednic, Douglas J McClure, Ludy Shih, Daniel Tarsy, Matt Welsh, and Paolo Bonato, "A web-based system for home monitoring of patients with parkinson's disease using wearable sensors," Biomedical Engineering, IEEE Transactions on, vol. 58, no. 3, pp. 831-836, 2011.

[5] Shyamal Patel, Bor-rong Chen, Thomas Buckley, Ramona Rednic, Doug McClure, Daniel Tarsy, Ludy Shih, Jennifer Dy, Matt Welsh, and Paolo Bonato, "Home monitoring of patients with parkinson's disease via wearable technology and a web-based application," in Engineering in Medicine and Biology Society (EMBC), 2010 Annual International Conference of the IEEE. IEEE, 2010, pp. 4411-4414.

[6] Karol J O’Donovan, Barry R Greene, Denise McGrath, Ross O'Neill, Adrian Burns, and Brian Caulfield, "Shimmer: A new tool for temporal gait analysis," in Engineering in Medicine and Biology Society, 2009. EMBC 2009. Annual International Conference of the IEEE. IEEE, 2009, pp. 3826-3829.

[7] Joseph P Giuffrida, David E Riley, Brian N Maddux, and Dustin A Heldman, "Clinically deployable kinesia technology for automated tremor assessment," Movement Disorders, vol. 24, no. 5, pp. 723-730, 2009.

[8] Giovanni Mostile, Joseph P Giuffrida, Octavian R Adam, Anthony Davidson, and Joseph Jankovic, "Correlation between kinesia system assessments and clinical tremor scores in patients with essential tremor," Movement Disorders, vol. 25, no. 12, pp. 1938-1943, 2010.
[9] Alberto J Espay, Paolo Bonato, Fatta B Nahab, Walter Maetzler, John M Dean, Jochen Klucken, Bjoern M Eskofier, Aristide Merola, Fay Horak, Anthony E Lang, et al., "Technology in parkinson's disease: Challenges and opportunities," Movement Disorders, 2016.

[10] S Arora, V Venkataraman, A Zhan, S Donohue, KM Biglan, ER Dorsey, and MA Little, "Detecting and monitoring the symptoms of parkinson's disease using smartphones: a pilot study," Parkinsonism \& related disorders, vol. 21, no. 6, pp. 650-653, 2015.

[11] Shyamal Patel, Konrad Lorincz, Richard Hughes, Nancy Huggins, John H Growdon, Matt Welsh, and Paolo Bonato, "Analysis of feature space for monitoring persons with parkinson's disease with application to a wireless wearable sensor system," in 2007 29th Annual International Conference of the IEEE Engineering in Medicine and Biology Society. IEEE, 2007, pp. 6290-6293.

[12] Yehezkel S Resheff, Shay Rotics, Roi Harel, Orr Spiegel, and Ran Nathan, "Accelerater: a web application for supervised learning of behavioral modes from acceleration measurements," Movement ecology, vol. 2, no. 1, pp. 1, 2014.

[13] Anestis Antoniadis, Xavier Brossat, Jairo Cugliari, and Jean-Michel Poggi, "Clustering functional data using wavelets," International Journal of Wavelets, Multiresolution and Information Processing, vol. 11, no. 01, pp. 1350003, 2013.

[14] Tom Hope, Avishai Wagner, and Or Zuk, "Clustering noisy signals with structured sparsity using time-frequency representation," arXiv preprint arXiv:1510.05214, 2015.

[15] Stephane G Mallat, "A theory for multiresolution signal decomposition: the wavelet representation," IEEE transactions on pattern analysis and machine intelligence, vol. 11, no. 7, pp. 674-693, 1989.

[16] Ingrid Daubechies, "Orthonormal bases of compactly supported wavelets," Communications on pure and applied mathematics, vol. 41, no. 7, pp. 909-996, 1988.

[17] John G Nutt, "Levodopa-induced dyskinesia review, observations, and speculations," Neurology, vol. 40, no. 2, pp. 340-340, 1990.

[18] Jae-Hoon Jun, Ji-Won Kim, Yuri Kwon, Gwang-Moon Eom, Seong-Beom Koh, Bongsoo Lee, Hyung-Sik Kim, Jeong-Han Yi, and Gye-Rae Tack, "Quantification of limb bradykinesia in patients with parkinsons disease using a gyrosensorimprovement and validation," International Journal of Precision Engineering and Manufacturing, vol. 12, no. 3, pp. 557-563, 2011. 